\title{
NFIRAOS: TMT facility adaptive optics with conventional DMs
}

Glen Herriot, Paul Hickson, B. L. Ellerbroek, David A. Andersen, T. Davidge, et al.

Glen Herriot, Paul Hickson, B. L. Ellerbroek, David A. Andersen, T. Davidge, D. A. Erickson, I. P. Powell, R. Clare, M. Smith, L. Saddlemyer, J.-P. Veran, "NFIRAOS: TMT facility adaptive optics with conventional DMs," Proc. SPIE 5903, Astronomical Adaptive Optics Systems and Applications II, 590302 (25 August 2005); doi: 10.1117/12.617849

SPIE. Event: Optics and Photonics 2005, 2005, San Diego, California, United States 


\title{
NFIRAOS: TMT facility adaptive optics with conventional DMs
}

\author{
Glen Herriot $^{\mathrm{a}}$, Paul Hickson ${ }^{\mathrm{b}}$, B. L. Ellerbroek ${ }^{\mathrm{c}}$, D.A. Andersen ${ }^{\mathrm{a}}$, T. Davidge ${ }^{\mathrm{a}}$, D. A. Erickson ${ }^{\mathrm{a}}$, I. P. \\ Powell $^{\mathrm{a}}$, R. Clare ${ }^{\mathrm{c}}$, M. Smith ${ }^{\mathrm{a}}$, L. Saddlemyer ${ }^{\mathrm{a}}, \mathrm{J}-\mathrm{P}$ Véran $^{\mathrm{a}}$, \\ ${ }^{a}$ National Research Council Canada - Herzberg Institute of Astrophysics (Canada) \\ ${ }^{\mathrm{b}}$ University of British Columbia (Canada) \\ ${ }^{\mathrm{c}}$ Thirty Meter Telescope Project, Pasadena CA
}

\begin{abstract}
Although many of the instruments planned for the TMT (Thirty Meter Telescope) have their own closely-coupled adaptive optics systems, TMT will also have a facility Adaptive Optics (AO) system feeding three instruments on the Nasmyth platform. For this Narrow-Field Infrared Adaptive Optics System, NFIRAOS (pronounced nefarious), the TMT project considered two architectures. One, described in this paper, employs conventional deformable mirrors with large diameters of about $300 \mathrm{~mm}$ and this is the reference design adopted by the TMT project. An alternative design based on MEMS was also studied, and is being presented separately in this conference. The requirements for NFIRAOS include 0.8-5 microns wavelength range, 30 arcsecond diameter output field of view (FOV), excellent sky coverage, and diffraction-limited atmospheric turbulence compensation (specified at $133 \mathrm{~nm}$ RMS including residual telescope and science instrument errors.) The reference design for NFIRAOS includes multiple sodium laser guide stars over a 70 arcsecond FOV, and an infrared tip/tilt/focus/astigmatism natural guide star sensor within instruments. Larger telescopes require greater deformable mirror (DM) stroke. Although initially NFIRAOS will correct a 10 arcsecond science field, it uses two deformable mirrors in series, partly to provide sufficient stroke for atmospheric correction over the 30 $\mathrm{m}$ telescope aperture, but mainly to partially correct a 2 arcminute diameter "technical" field to sharpen near-IR natural guide stars and improve sky coverage. The planned upgrade to full performance includes replacing the groundconjugated DM with a higher actuator density, and using a deformable telescope secondary mirror as a "woofer." NFIRAOS incorporates an instrument rotator and selection of three live instruments: a near-Infrared integral field Imaging spectrograph, a near-infrared echelle spectrograph, and after upgrading NFIRAOS to full multi-conjugation, a wide field (30 arcsecond) infrared camera.
\end{abstract}

Keywords: TMT, Thirty Meter Telescope, adaptive optics, NFIRAOS

\section{INTRODUCTION}

The Thirty Meter Telescope (TMT) project is a public-private partnership that fulfills the goals of a concept called the Giant Segmented Mirror Telescope (GSMT), which was identified in the National Academy of Sciences report "Astronomy and Astrophysics in the New Millennium" as the highest-priority new ground-based facility for the first decade of the 21st century. The TMT project is a collaboration of Caltech, University of California (UC), the Association of Universities for Research in Astronomy (AURA), and the Association of Canadian Universities for Research in Astronomy (ACURA). The goal of the TMT project is to construct an extremely large telescope based on over 700 hexagonalshaped mirror segments that stretch a total of 30 meters in diameter. Such a telescope also needs adaptive optics systems that compensate for natural distortions of the incoming light by Earth's atmosphere and huge science instruments containing dozens of mirrors, detectors, and complex filters. The TMT will gather light in visible and infrared wavelengths to help astronomers answer the most challenging questions in astronomy and astrophysics, from "How do planets form?" to "What is the ultimate fate of our galaxy?" The founding members of the TMT formalized their partnership in June 2003, with the goal of beginning full science operations of the telescope as early as 2015, on a site to be chosen in 2007. Funding for the project is expected to be a mix of public and private contributions, with a significant fraction of open community access to the resulting observing time for astronomers in the United States and Canada.

Astronomical Adaptive Optics Systems and Applications II, edited by Robert K. Tyson, Michael Lloyd-Hart, Proceedings of SPIE Vol. 5903, 590302, (2005) · 0277-786X/05/\$15 · doi: 10.1117/12.617849 


\subsection{The Thirty Meter Telescope Project}

The Thirty Meter Telescope (TMT) Project (See www.tmt.org) is currently in the design and development phase. A reference design for the telescope has been developed with the following key features:

- $\quad$ A 30 meter $\mathrm{f} / 1$ filled aperture primary with more than 700 hexagonal mirror segments, each actively controlled with edge sensors and 3 actuators providing tip/tilt/piston compensation.

- $\quad$ An $\mathrm{f} / 15$ final focus.

- $\quad$ A Gregorian secondary mirror (M2) approximately $3.6 \mathrm{~m}$ in diameter.

- $\quad$ A field of view of 20 arcminutes.

- $\quad$ The elevation axis in front of the primary mirror

- $\quad$ Large Nasmyth platforms fed by an articulated tertiary mirror (M3)

- $\quad$ Wavelength coverage from 0.31 to 28 microns

- $\quad$ Operational zenith angle range from 1 to 65 degrees from zenith

- $\quad$ Both seeing limited and adaptive optics observing modes

TMT will be commissioned with a rigid secondary mirror. However, as soon as practical after first light, it is planned to upgrade to an adaptive secondary mirror.

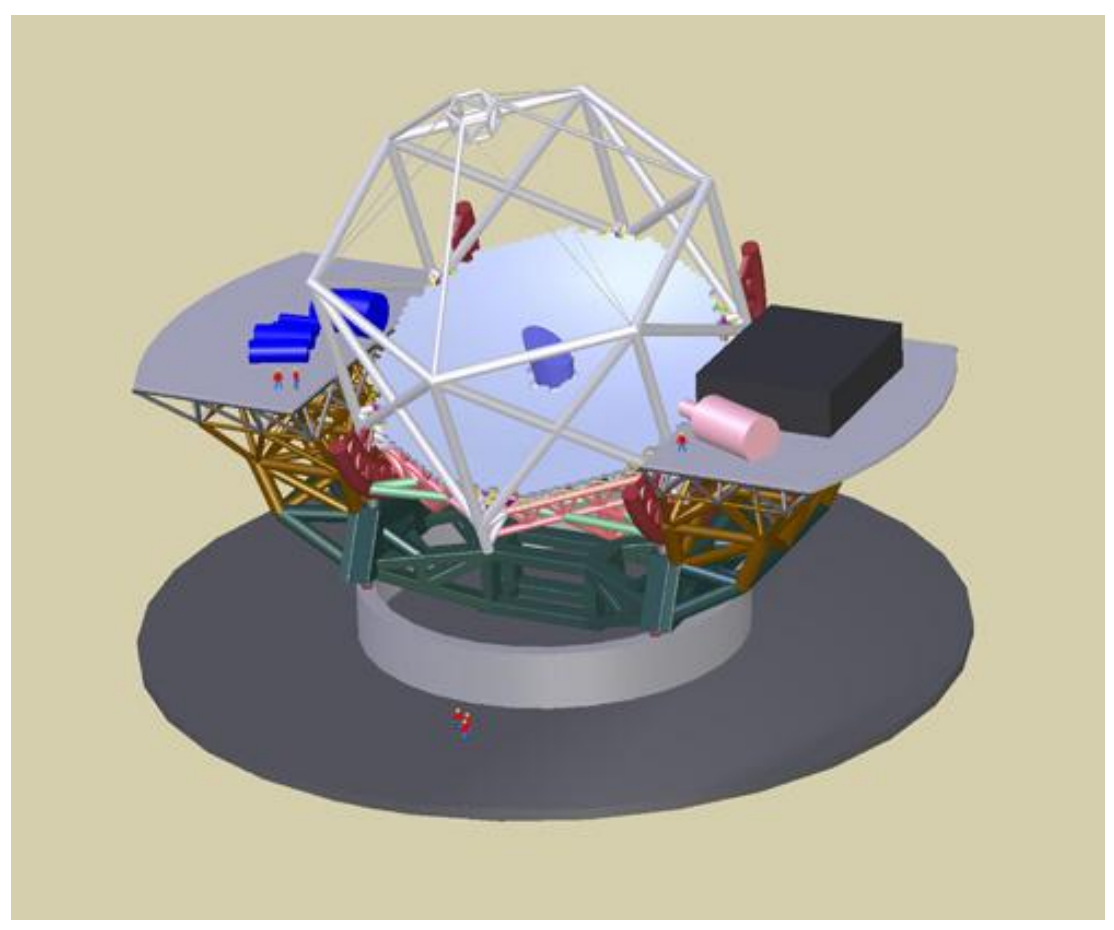

\section{Figure 1 Thirty Meter Telescope with instruments on Nasmyth platforms}

Sites in both hemispheres are under consideration, with a vigourous site survey programme underway to assess key environmental parameters.

\subsection{TMT AO Program}

NFIRAOS must feed a suite of instruments, work seamlessly with the observatory, and preserve flexibility for the future. It is part of the overall TMT program for adaptive optics managed by Brent Ellerbroek of the TMT project office in Pasadena. The TMT instrument program is managed by David Crampton, NRC-HIA in Victoria. The adaptive optics program includes component development projects and feasibility studies for items like deformable mirrors, wave-front sensor (WFS) detectors, real time computers, and adaptive secondary mirrors. As well, feasibility studies of several 
specialized AO systems, intimately married to specific science instruments are underway. See the Ellerbroek paper in this conference.

\begin{tabular}{|l|l|l|l|}
\hline \multicolumn{2}{|c|}{ AO System } & \multicolumn{2}{c|}{ Feeding Instrument } \\
\hline NFIRAOS & $\begin{array}{l}\text { Narrow Field Infra-red adaptive optics } \\
\text { system. }\end{array}$ & IRIS & Integral Field Spectrograph 1- 2.5 $\mu \mathrm{m}$ \\
\cline { 3 - 4 } & & NIRES & Near infrared Echelle spectrograph 1-5 $\mu \mathrm{m}$ \\
\cline { 2 - 4 } & WIRC & Wide field (30") near-infrared imager 1- 5 $\mu \mathrm{m}$ \\
\hline MIRAO & Mid-infrared adaptive optics & MIRES & Mid-infrared Echelle Spectrograph 5-28 $\mu \mathrm{m}$ \\
\hline ExAO & Extreme Contrast adaptive optics & PFI & Planet formation imager \\
\hline MOAO & $\begin{array}{l}\text { Multi-object adaptive optics. Open-loop } \\
\text { correction of individual science fields } \\
\text { within 5-arcminute field of view. }\end{array}$ & IRMOS & $\begin{array}{l}\text { Infrared multi-object spectrograph. Multiple } \\
\text { deployable IFU spectrograph 1- 2.5 } \mu \mathrm{m}\end{array}$ \\
\hline
\end{tabular}

Table 1 TMT AO \& Instruments

\subsection{Narrow-Field Infrared Adaptive Optics System, NFIRAOS}

Currently, the National Research Council of Canada's Herzberg Institute of Astrophysics is contracted to conduct a conceptual design study for NFIRAOS, in close collaboration with the TMT project office. This concept study ends with a conceptual design review in April 2006. There is only a single conceptual study for NFIRAOS, which is planned to stay slightly ahead of instruments' design and development throughout the project. Because it is a key first-light facility of TMT, modelling, error budget, and interfaces are directed from the project office. The TMT Project will develop \& supply detectors, DMs, high-speed electronics to NFIRAOS. The conceptual study will provide costing estimates for NFIRAOS as input to the overall TMT Costing Review in the 3rd quarter of 2006.

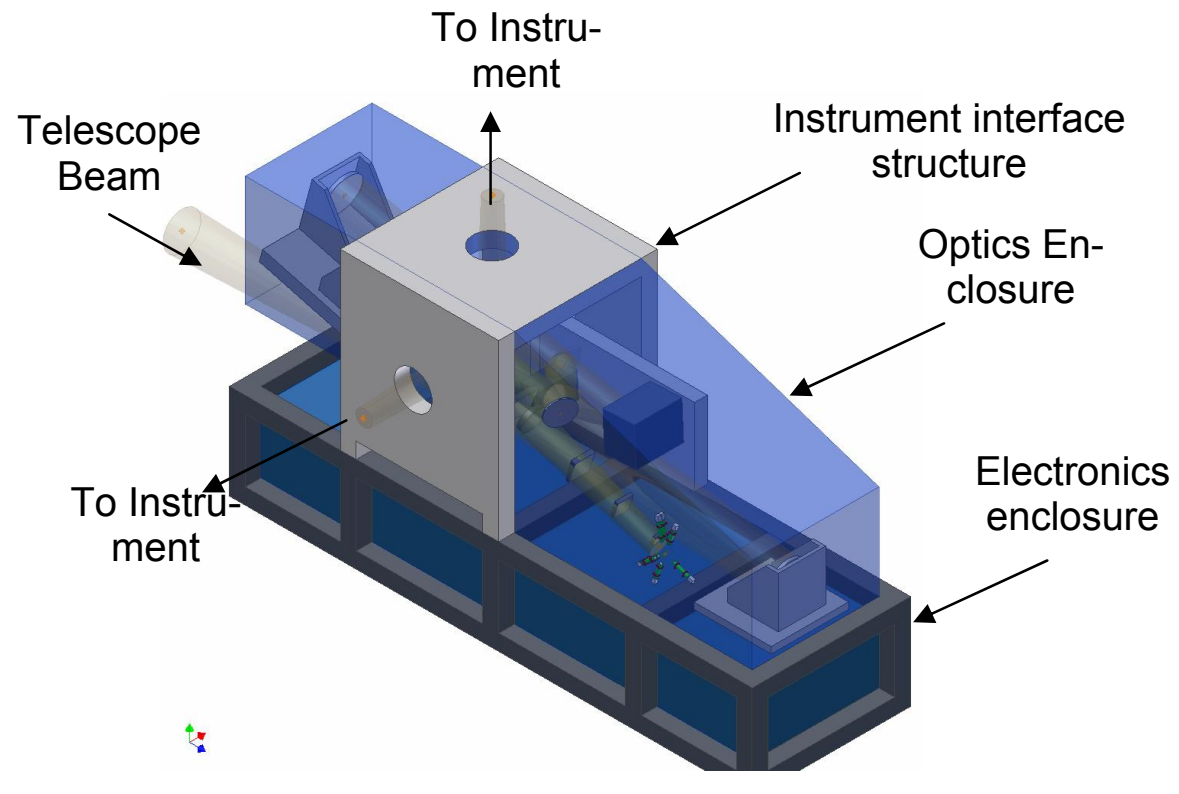

Figure 2 NFIRAOS Layout

NFIRAOS will be the main facility adaptive optics system for TMT. NIFIRAOS will provide near diffraction-limited compensation of atmospheric turbulence using laser guide star adaptive optics. It will have opto-mechanical interfaces with three narrow-field (10" - 30"), near infra-red science instruments and reside on the TMT Nasmyth platform. It has software and control interfaces with the Observatory Control, Telescope Control, and Data Handling Systems. Its adaptive optics control functions will be integrated with the Laser Guide Star Facility, the Secondary Mirror Control System, 
and on-instrument wavefront sensors included within science instruments. To maximize sky coverage, these sensors will detect near infra-red. Instruments will have either multiple natural guide star tip/tilt sensors, or single tip/tilt/focus/astigmatism (i.e. $2 \times 2 \mathrm{SH}$ ) wavefront sensors. The current plans are to deliver a baseline NFIRAOS that corrects a 10 arcsecond field for astronomy, but which also moderately corrects a 2-arcminute diameter technical field for this natural guide star wavefront sensing. NFIRAOS will be also be upgradeable to a multi-conjugate AO system that corrects a wider science field ( 30 arcsecond) for a near infra-red science instrument, planned to be an imager.

\section{NFIRAOS FUNCTIONALITY \& PERFORMANCE}

Figure 3 shows part of TMT's Nasmyth platform with NFIRAOS accepting an f/15 input beam from the telescope and directing the corrected light to one of three instruments. Switching between instruments shall require no more than 10 minutes during nighttime observing.

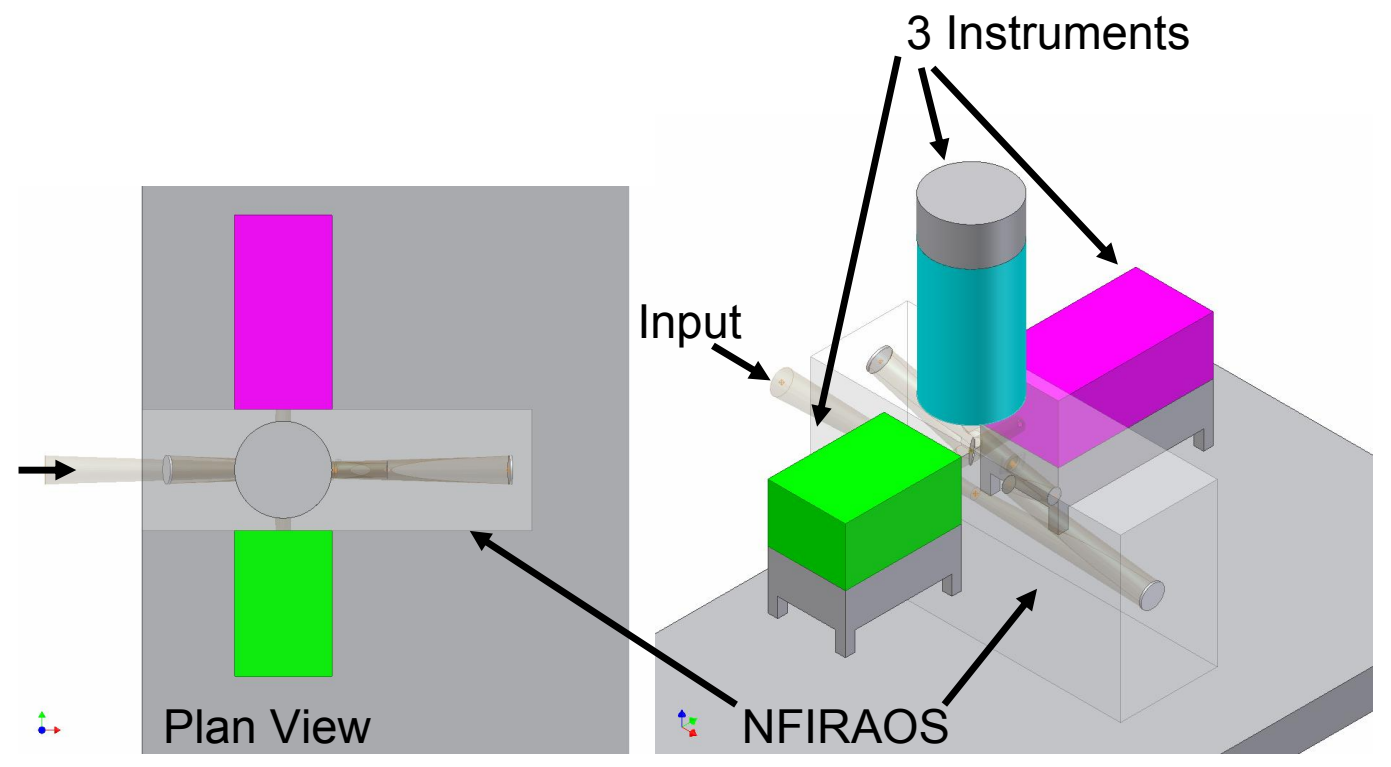

Figure 3 NFIRAOS shown on part of Nasmyth platform with three instruments

NFIRAOS is intended principally for laser guide star operation, to provide $50 \%$ sky coverage at the galactic pole with near-diffraction limited performance, defined, under nominal seeing of $r_{0}=0.15 \mathrm{~m}$ in the observing direction, to be 120 $\mathrm{nm}$ rms wavefront error on-axis, and $133 \mathrm{~nm}$ rms over a thirty arcsecond field of view. This latter field of view and level of image quality is not expected at first light, but after upgrading the deformable mirrors. Furthermore it assumes an isoplanatic angle $\left(\theta_{0}\right)$ of 2.5 arcsecond, which is an estimate before substantial site survey data has been obtained.

The technical, or patrol field for Natural guide star sensing is sufficiently large (currently 2 arcminutes diameter) to achieve the required sky coverage because NFIRAOS relies on moderate image sharpening (expected Strehl $\sim 0.1$ ) of near-IR natural guide stars by the two deformable mirrors. To that end, the control system, while optimized over the science field, operates as "MCAO-lite" to improve the measurement precision of the tip/tilt wavefront sensors. This entire technical field is fed to instruments for natural star wavefront sensing.

NFIRAOS-fed instruments will include natural guide star (NGS) wavefront sensor(s) to provide fast guiding, calibrate focus biases in the laser guide star (LGS) WFS induced by variations in the range to the sodium layer, and also detect quadratic modes of focal anisoplanatism. The magnitude limit and integration time for this sensor(s) will be consistent 
with 50 per cent sky coverage at the galactic pole. Thus, typical operation will use LGS WFS within NFIRAOS blended with measurements from NGS IR wavefront sensors within instruments.

It is proposed that instrument builders provide their choice of natural guide star sensing via either of

- a single $2 \times 2$ Shack-Hartmann wavefront sensor

- multiple tip/tilt sensors

The first technique detects tip, tilt, focus and astigmatism, to break the degeneracy caused by atmospheric quadratic modes imperfectly sensed by laser guide stars. This uncertainty would cause image distortion, such as magnification errors and differential XY magnification. The latter multiple NGS approach choice directly senses tip/tilt at three stars throughout the field, perhaps by sub-array readout of an imaging detector. It avoids possible vignetting by pickoff mirrors or losses due to dichroics, but as well as having poorer observing efficiency from acquiring multiple natural stars, it is less resistant to focus errors due to uncertainty in the altitude of the sodium layer. See section 5.

NFIRAOS will use 5-9 Sodium laser guide stars with good beam quality (on the sky) and with a total power of $\sim 100 \mathrm{~W}$ initially and $\sim 400 \mathrm{~W}$ for the MCAO upgrade. The LGS WFSs will operate with a mean guide star range from 85 to 200 $\mathrm{km}$ and NFIRAOS will function at zenith angles from 0 to 60 degrees, and with values of $\mathrm{r}_{0}$ (in the direction of the observation) as small as $0.10 \mathrm{~m}$ at $\lambda=0.5 \mu \mathrm{m}$. NFIRAOS is required to operate without undue DM saturation or control instabilities. Thus TMT will procure deformable mirrors with stroke amplitude six times the RMS actuator command necessary for turbulence compensation ( $5 \sigma$ for atmosphere and $1 \sigma$ for margin.)

\subsection{Calibration facilities}

NFIRAOS will include remotely deployable simulated NGS sources, and simulated LGS sources. These devices will be used to characterize and calibrate system performance, system alignment, deformable mirror to wavefront sensor influence-functions, WFS detectors, and non-common path aberrations between the wavefront sensing and science optical paths. There will also be a manually inserted atmospheric turbulence simulator at the input focal plane. As a goal, the turbulence simulator will include multiple phase screens in planes conjugate to ranges between 0 and $10 \mathrm{~km}$. NFIRAOS will also be usable with simulated NGS and LGS sources located at the prime focus of the telescope.

NFIRAOS will compensate in real-time for variations in both NGS and LGS WFS gains and biases induced by changes in seeing, flexure and thermal effects and variations in the sodium layer density profile.

NFIRAOS-fed instruments will include a "truth" sensor to measure and characterize loop performance on bright stars. For use without lasers, NFIRAOS-fed instruments will include a high-order NGS WFS to control the DMs. [This sensor might be the high-order NGS truth WFS, but operated at higher sample rate.]

NFIRAOS will provide performance estimates usable for characterizing the AO-compensated point spread function (PSF) at the science instrument focal plane. As a goal, these estimates should be available in near-real-time in a format usable for optimizing the performance of the AO control loop.

\subsection{Technical risk avoidance}

NFIRAOS will minimize dependence upon unproven AO component technologies. Thus, it will be operated with currently demonstrated guide star laser pulse formats and piezostack deformable mirrors with actuator stroke and actuator density similar to existing mirrors.

As well, NFIRAOS will use NGS and LGS wavefront sensors that are minimal extrapolations from existing detector technology in terms of read noise, pixel read rate, and number of pixels. However, we hope to use a novel radial format CCD for LGS sensing to help mitigate up to 4 arcseconds of spot elongation on outer subapertures. Rectangular subarrays, notionally $16 \times 4$ pixels, will be placed non-contiguously across the CCD at each lenslet image, with the subarrays' long axes aligned with the elongated laser spot. This geometry minimizes the number of pixels read out, but provides sufficient sampling to permit spot centroiding via correlation tracking or matched filtering, benefiting from structure in the sodium layer. 


\subsection{Upgrade Path to MCAO}

Recall that even the baseline 10" field of view NFIRAOS operates as a modest MCAO system, to improve sky coverage. During this phase, it is expected that the TMT secondary mirror will be a rigid body with little tip/tilt bandwidth. The baseline NFIRAOS will have the following two DMs.

1) A ground-conjugated DM with 60 actuators across the $300 \mathrm{~mm}$ pupil, but with additional rings of unilluminated actuators filling a $360 \mathrm{~mm}$ diameter circle. These actuators will have a stroke of at least $9 \mu \mathrm{m}$. This mirror will be mounted on a tip/tilt platform with a $20 \mathrm{~Hz}$ tip/tilt bandwidth (and a goal of $40 \mathrm{~Hz}$.) This specification is for the tip/tilt platform while tilting the DM, not the overall closed loop bandwidth of the control system. See modeling details in section 5 for control bandwidth estimates.

2) A DM conjugate to $12 \mathrm{~km}$, with 60 actuators across the $360 \mathrm{~mm}$ beamprint of the 2 arcminute technical (NGS WFS) field of view.

The upgrade to a 30 " field corrected by Multi-conjugate adaptive optics is planned as follows:

1) Remove the ground-conjugated DM from the tip/tilt platform and discard it.

2) Install, at the conjugate to $0 \mathrm{~km}$, a new DM with a $<3 \mu \mathrm{m}$ stroke and 120 actuators across the pupil.

3) Upgrade the LGS WFS cameras to 120 subapertures across the pupil.

4) Replace the telescope secondary mirror with an adaptive secondary to act as a "woofer" for the new DM.

5) Quadruple the laser power.

\section{OPTOMECHANICS}

The input beam from the telescope is $2 \mathrm{~m}$ above the Nasmyth platform, and focuses $5 \mathrm{~m}$ beyond the edge of the primary mirror. The output $\mathrm{f} / 15$ beam from NFIRAOS comes to a focus $750 \mathrm{~mm}$ beyond the side ports' instrument mounting faces, and $600 \mathrm{~mm}$ above the upper port face. However, instrument snouts may intrude into NFIRAOS, providing $1 \mathrm{~m}$ back focal distance in every case. NFIRAOS includes an input window, and an airtight seal with each science instrument's input window, and light-tight shutters at each window.

The side ports are $3 \mathrm{~m}$ above the platform. Image de-rotation will be done within the side-facing narrow-field instruments by either a " $\mathrm{K}$ " mirror assembly, or by rotating the instrument about a horizontal axis. NFIRAOS provides a mounting face for an instrument rotator bearing at the top port. Notionally this is earmarked for WIRC, the 30' imaging camera.

Very preliminary estimates of masses are as follows: NFIRAOS $10-15$ T, WIRC 10 T, IRIS 4 T, and NIRES 8 tonnes. NFIRAOS itself has dimensions $8.2 \times 4.2 \times 2.5$ meters, but these will likely grow as the design is refined.

The science wavelength range is 1.0-5.0 $\mu \mathrm{m}$, with a goal of 0.6-5.0 $\mu \mathrm{m}$. The optical throughput over this wavelength range should exceed $75 \%$. The "Baseline" output science field of view is a 10 arcsecond square. The output science field will be corrected over a 30 arcsecond square for the MCAO "Upgrade." The technical field of view (for 1.0-2.5 $\mu \mathrm{m}$ natural guide star sensing) delivered to instruments is 2' diameter.

The optical design images the TMT secondary mirror at the ground-conjugate NFIRAOS deformable mirror. The design incorporates a high-order 60x60 piezostack DM, in series with a low-order (30-60 across pupil), large stroke "woofer" DM. The "woofer" is placed in a plane optically conjugate to $\mathrm{h}=12 \mathrm{~km}$ to simplify the MCAO upgrade. The on-axis beam diameter is $0.30 \mathrm{~m}$ at both mirrors so that they may be in single optical relay.

Figure 4 on the left shows the science optical path. Light from the telescope is collimated by an off-axis parabola, reflects off the high altitude, and then ground conjugated DMs, passing through a beamsplitter before being reimaged by a matching OAP. Finally a steering mirror diverts the corrected light to one of three instrument ports.

Figure 5 shows the laser guide star optics. A short-wave reflecting beamsplitter send the laser light off a fold and then to a replica of the off-axis parabola OAP2. A zoom collimator refocuses the sodium layer while compensating for aberrations due to the finite and varying range distance. The laser beacons focus on a facetted mirror that acts as a field stop and diverts each beacon into its own stationary LGS camera with collimator, lenslet array and CCD. 

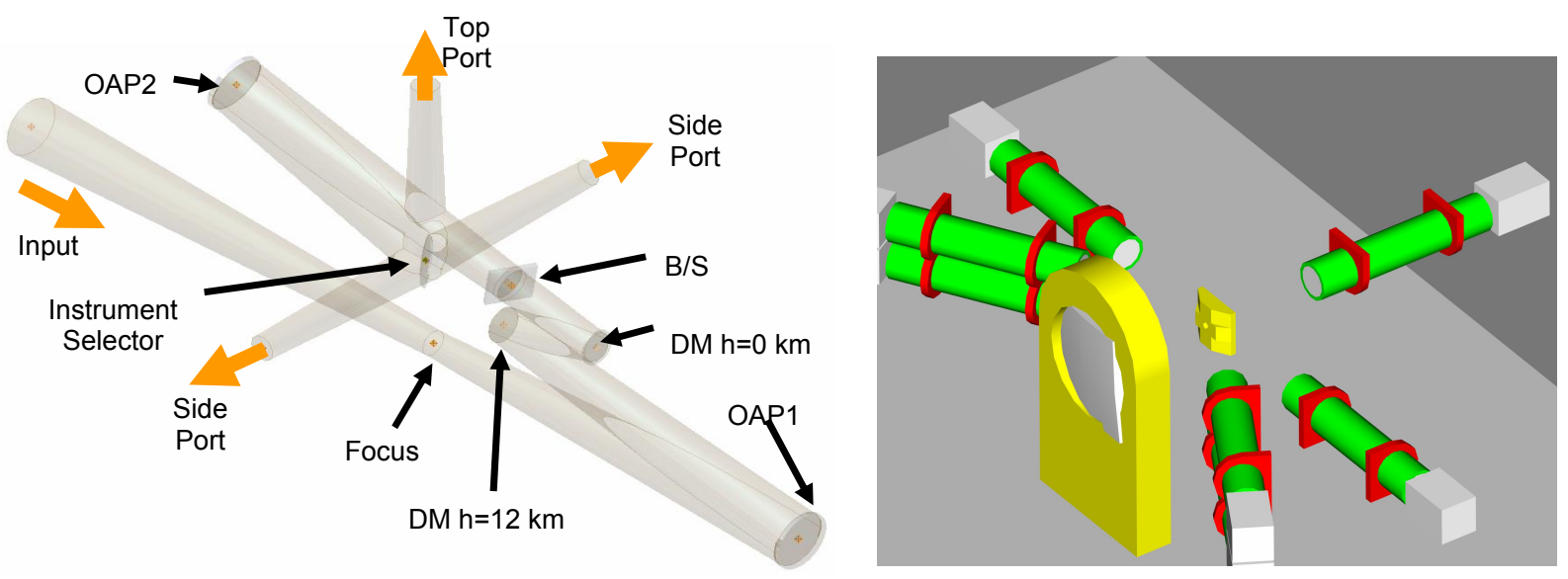

Figure 4 Left: Science Path. Right: LGS WFSs cameras facing facetted mirror after last element of zoom

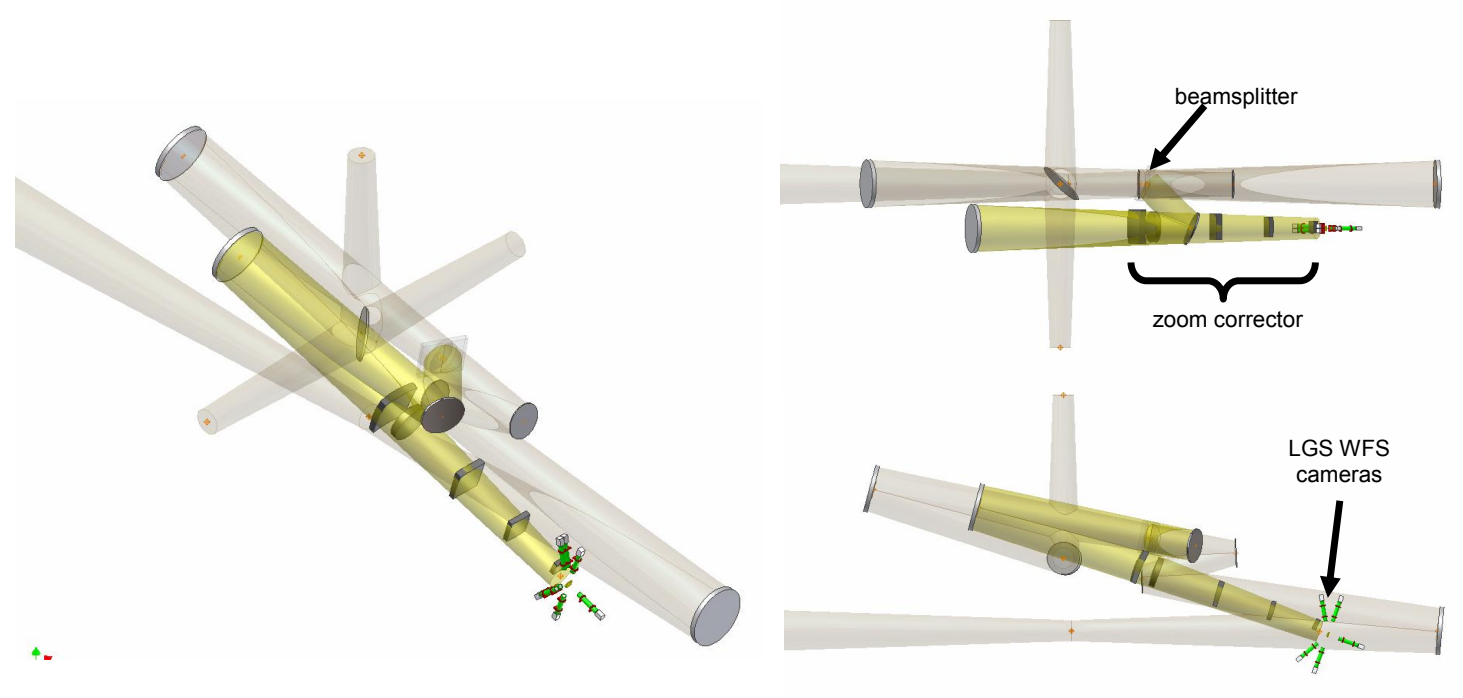

Figure 5 Laser Guide star optics Left: isometric, Upper Right: plan view, Lower Right Side view.

These optomechancal layouts have been done by Darren Erickson (mechanics) and Ian Powell (optics), starting from a previous optical design by Dick Buchroeder.

\subsection{Commissioning Camera}

For integration and testing of NFIRAOS, there will be a commissioning camera, consisting of an IR-sensitive NGS WFS, and a small instantaneous field patrolling IR imager, available to be attached to one of the three NFIRAOS output ports. This will probably reside on the top port, which is intended for the imager, a second generation instrument. 


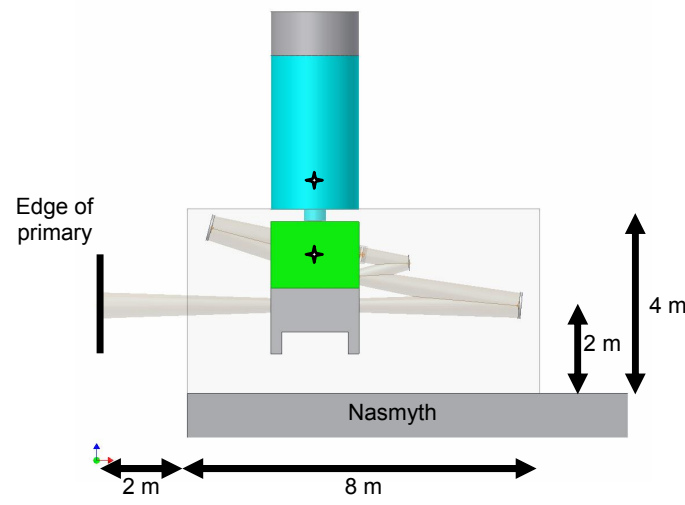

Figure 6 Side View showing NFIRAOS and three instruments

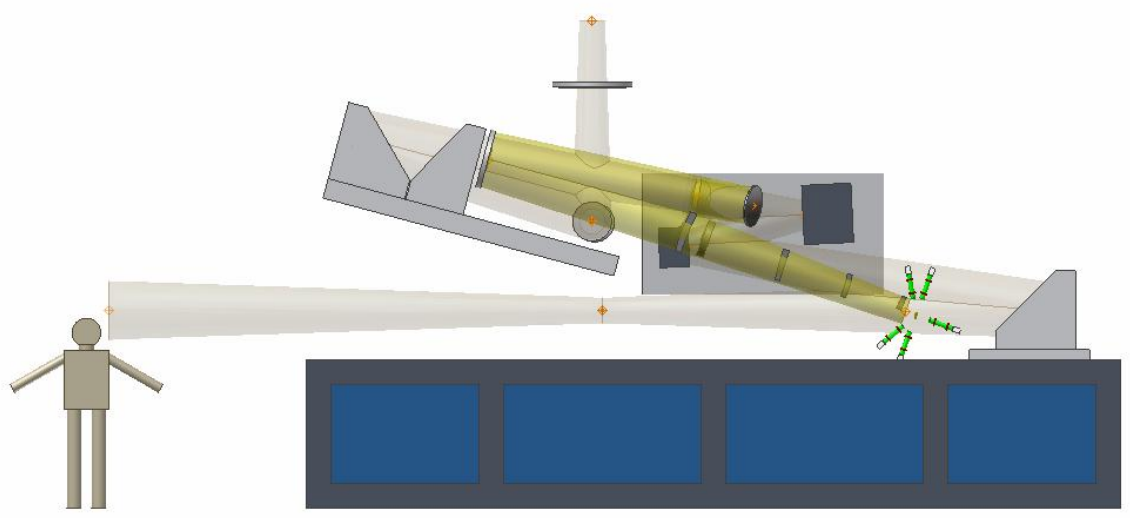

Figure 7 Side view of Science and LGS optics above electronics cabinets

\section{EMISSIVITY AND REFRIGERATION}

The overall TMT science requirements demand that NFIRAOS' inter-OH background in K band not exceed $15 \%$ of the background due to the sky and the 3-mirror telescope at a nominal temperature of $273 \mathrm{~K}$. The intent is that a real NFIRAOS will only increase integration time on the sky by $15 \%$. With 5 mirrors, a beamsplitter, and a double-pane entrance window, NFIRAOS' emissivity is $20 \%$. To meet the background specification requires cooling of all optical surfaces to -33 Celsius.

The jagged upper curve in the left panel of Figure 8 shows the background requirement, calculated by adding a greybody emission, representing the telescope, to K-band sky background data courtesy of the Gemini Observatory. Fifteen per cent of this total at each wavelength in $\mathrm{K}$ band is the not-to-exceed specification for NFIRAOS. The lower smooth curve on the left is the emission from NFIRAOS, for the temperature where this background just kisses the specification curve, (circled). The right hand panel shows how the required temperature would vary versus NFIRAOS emissivity.

Clearly, this temperature range, together with the mass and volume of NFIRAOS will make both servicing it and changing instruments challenging. Humidity from personnel will condense on critical surfaces, yet warming it above this elevated dew point will result in very long cycle times. 


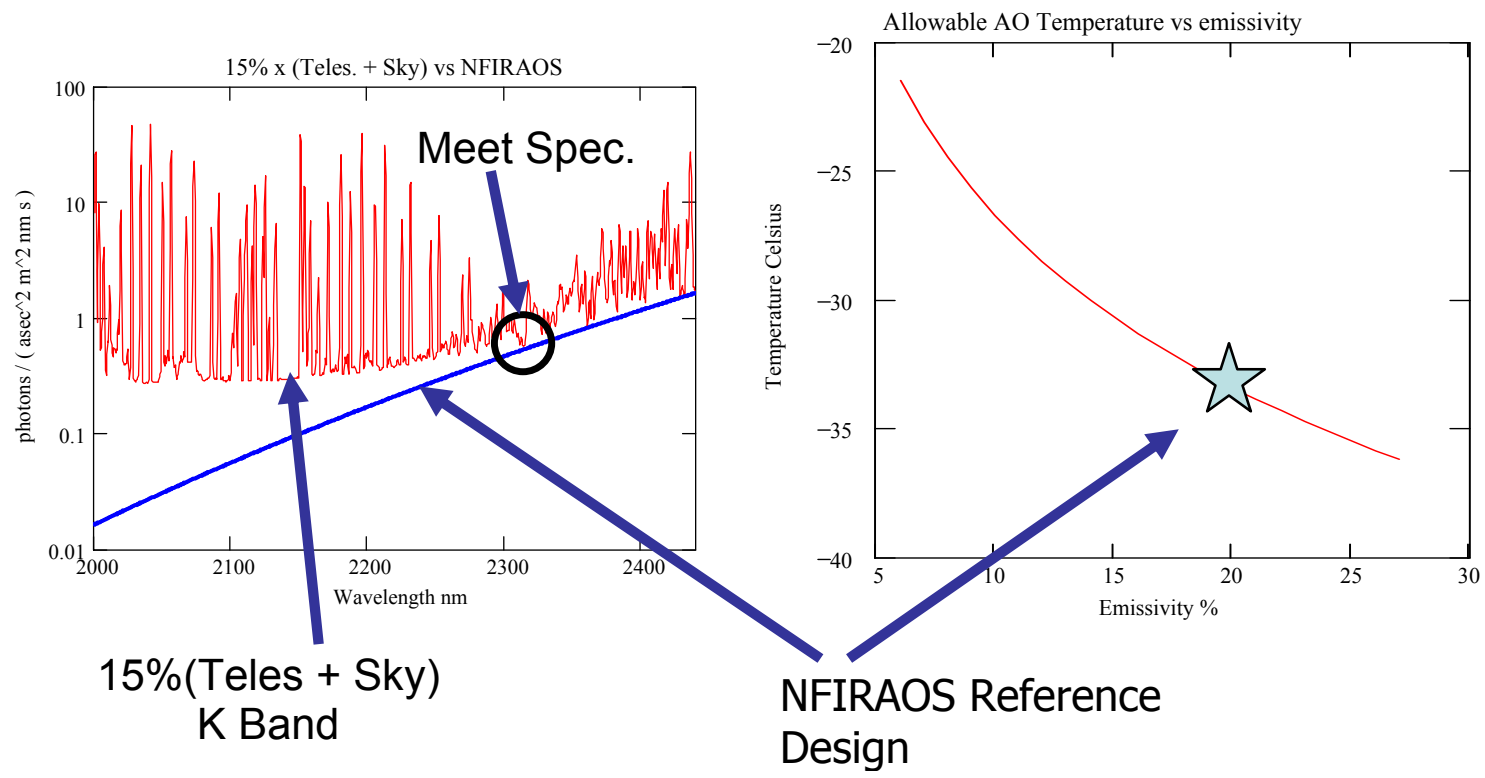

Figure 8 Enclosure Temperature to meet background specification

\section{PERFORMANCE MODELLING}

\subsection{Tip tilt and sky coverage}

Richard Clare of the TMT Project Office is studying the sky coverage and tip tilt residual errors for a variety of parameters:

- $\quad$ Number of NGS: $1-5$ if T/T only

o or $1 \mathrm{~T} / \mathrm{T} / \mathrm{F} / \mathrm{A}$ sensor

$\circ$ or $1 \mathrm{~T} / \mathrm{T}$ sensor plus a Rayleigh beacon

- bandwidth:

○ sampling $\sim 100-1000 \mathrm{~Hz}$

- $-3 \mathrm{db}$ cutoff of tip/tilt mirror ranging from 10 to $100 \mathrm{~Hz}$

- read noise: TBD 1, $510,15 \mathrm{e}-$

- windshake: possible 3-5 Hz telescope resonance inducing $\mathrm{T} / \mathrm{T} / \mathrm{F} / \mathrm{Coma}$ of uncertain amplitude

- $\quad$ performance metrics: rms tip/tilt jitter, quadratic mode residuals)

The initial conclusion is that, based on median wind speeds and early structural and wind buffeting analysis for the telescope, and given the tip/tilt mirror $-3 \mathrm{~dB}$ bandwidth of $20 \mathrm{~Hz}$, the residual telescope windshake after correction by NFIRAOS approximately equals the entire NFIRAOS error budget. The DM conjugate to the ground does not have enough stroke to correct tip/tilt and high order aberrations simultaneously. We will explore using the $\mathrm{h}=12 \mathrm{~km} \mathrm{DM}$ as a tweeter to correct the tip/tilt high temporal frequencies. No other surface is small and/or close to a ground conjugate.

\subsection{Order of correction, location of DM}

Meanwhile, Jean-Pierre Véran and David Andersen, are evaluating the tradeoffs among quantity and location of laser guide stars, altitude and actuator density of the higher DM, with a performance metric of tilt-removed wavefront error. Interestingly, the best performance results from putting 5-7 lasers in a 35" radius ring, without a central beacon.

- $\quad$ Order of correction of high-altitude DM, e.g. 30x30 to 60x60

- DM conjugate options $9-14 \mathrm{~km}$

- LGS asterism options, 5 -9 sodium beacons 
- loop bandwidth sampling 0.5 to $2 \mathrm{kHz}$

- WFS measurement noise TBD 2 to $10 \mathrm{e}-$

Remaining important higher-order effects and implementation issues to be studied include.

- Wavefront sensor modeling (LGS elongation, partially sharpened NGS)

- Deformable mirror modeling (hysteresis, dynamic range, influence functions)

- Telescope interactions (M1 segmentation, M1/M2/Nasmyth windshake)

- AO implementation errors (misregistration, NCPA, ...)

- Advanced algorithms and adaptive background tasks (woofer/tweeter control, pseudo open-loop control, uncontrolled mode removal, loop and centroid gain estimation, clipping)

\section{SOFTWARE INTERFACES}

NFIRAOS interfaces with the TMT Observatory Control System (OCS) for overall sequencing of observational steps involving NFIRAOS, science instruments, and the LGS projection facility. NFIRAOS offloads wavefront corrections to the TMT Telescope Control System (TCS) to compensate for telescope pointing errors, primary mirror figure errors, and secondary mirror decenter, focus (and higher order for AM2 upgrade) errors, and retrieves the telescope pointing vector. TMT Data Handling System (DHS) stores WFS measurements and DM actuator commands as needed to characterize performance and estimate the AO-compensated PSF at the science instrument. NFIRAOS connects to the TMT Secondary Control System (SCS) for high-bandwidth control of secondary mirror tip/tilt if the secondary mirror is used for this function or for high bandwidth mirror figure control if and when an adaptive secondary mirror is implemented. NFIRAOS communicates with the TMT LGS Facility (LGS Facility) for pointing control of laser guide stars on the sky, synchronizing LGS WFS measurements with laser pulses (if a pulsed laser is used), and to be notified of interruptions to LGS projection. NFIRAOS receives tip/tilt/focus measurements from any available on-instrument wavefront sensors in science instruments.

\begin{tabular}{|l|l|l|}
\hline System & Commands/Inputs from NFIRAOS & Commands/Outputs to NFIRAOS \\
\hline $\begin{array}{l}\text { Observatory Control } \\
\text { System }\end{array}$ & AO control loop status & $\begin{array}{l}\text { Commands to start/stop/adjust AO loop op- } \\
\text { eration }\end{array}$ \\
\hline $\begin{array}{l}\text { Telescope/Mount } \\
\text { Control System }\end{array}$ & $\begin{array}{l}\text { Pointing offloads } \\
\text { Focus and other low order M1 figure ad- } \\
\text { justments }\end{array}$ & Telescope pointing state \\
\hline $\begin{array}{l}\text { Secondary Control } \\
\text { System }\end{array}$ & $\begin{array}{l}\text { Tip/tilt and focus commands } \\
\text { Actuator commands (in the case of an adap- } \\
\text { tive secondary) }\end{array}$ & Secondary mirror actuator state \\
\hline LGS Facility & $\begin{array}{l}\text { Commands to start/stop/adjust laser opera- } \\
\text { tion } \\
\text { Commands to start/stop/adjust LGS projec- } \\
\text { tion and LGS control loops } \\
\text { AO control loop status (in response to re- } \\
\text { quests from laser safety systems) }\end{array}$ & Laser system status \\
LGS projection system status \\
Halt/stop commands due to laser traffic con- \\
trol blocks or safety alarms
\end{tabular}




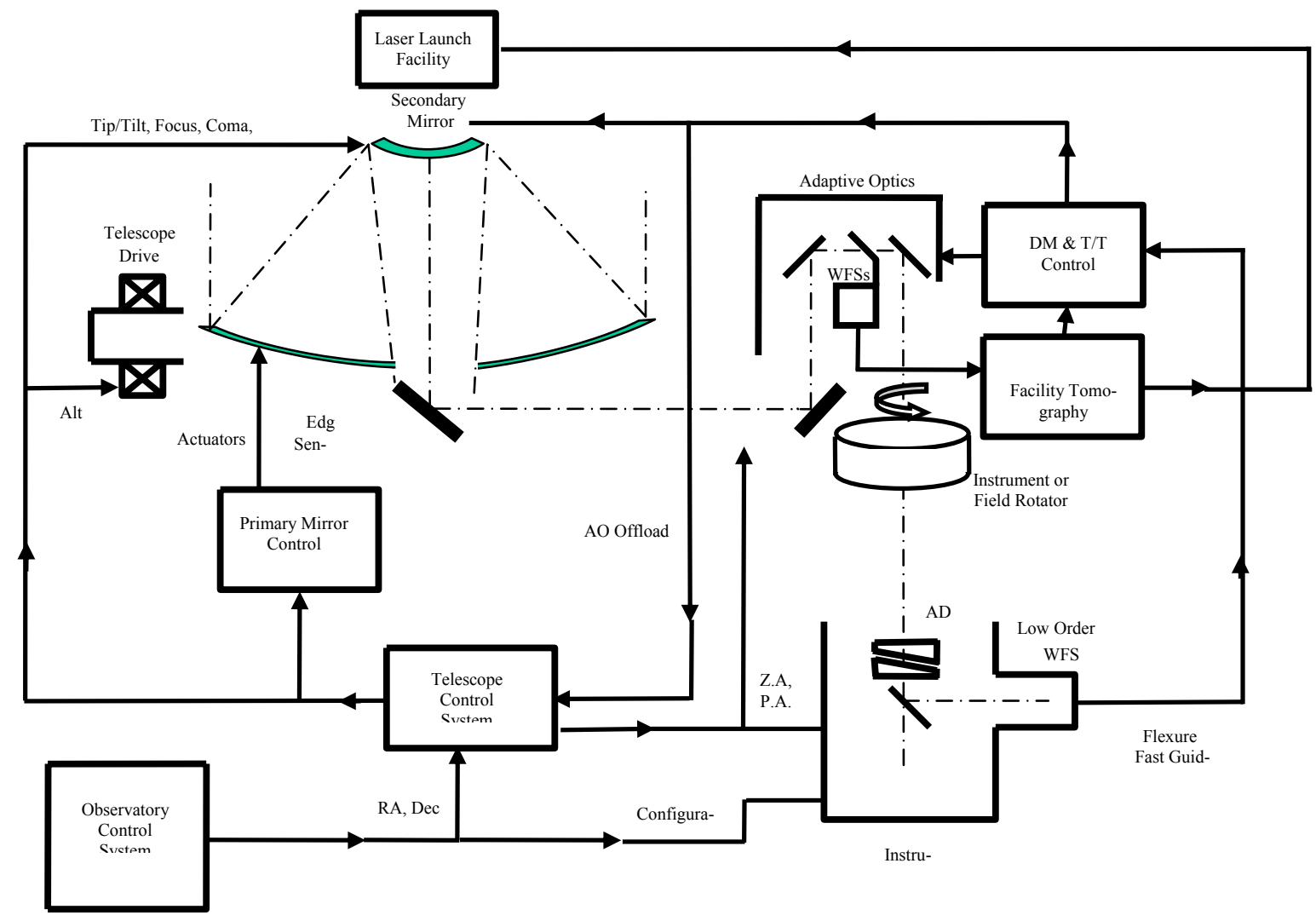

Figure 9 Software Interfaces Data Flow

\section{CONCLUSIONS}

NFIRAOS, the facility Near-Infrared Adaptive Optics System for the Thirty Metre Telescope, resides in a cooled enclosure on the telescope Nasmyth platform. It employs 5-7 laser guide stars and conventional piezostack deformable mirrors to feed three instruments. Initially it will correct a 10 arcsecond scientific field, but will be upgraded to a multiconjugate adaptive optics system correcting a 30 arcsecond field of view. NFIRAOS is intimately connected with the observatory control system to ensure efficient observing.

\section{REFERENCES}

1. Tom Geballe, Phil Puxley http://www.gemini.edu/sciops/ObsProcess/obsConstraints/atmmodels/nearIR_skybg_16_15.dat

\section{ACKNOWLEDGEMENTS}

The authors present this paper as part of the work of the Thirty Meter Telescope (TMT) Project. TMT is a partnership of the Association of Universities for Research in Astronomy (AURA), the Association of Canadian Universities for Research in Astronomy (ACURA), the California Institute of Technology and the University of California. The partners gratefully acknowledge the support of the Gordon and Betty Moore Foundation, the US National Science Foundation, the National Research Council of Canada (NRC), the Gemini Partnership and the Natural Science and Engineering Research Council Canada (NSERC). 